\title{
An overview on fluoroquinolone drugs for the treatment of tubercular infection
}

\begin{abstract}
The fluoroquinolones levofloxacin, moxifloxacin and gatifloxacin have potent bactericidal activities against Mycobacterium tuberculosis. They have the potential activity in managing both drug-susceptible and drug-resistant tuberculosis as well as the possibility of shortening the period of therapy. The emergence of drug-resistance, fluoroquinolone-resistant, multidrug-resistant and extensively drug-resistant tuberculosis created a challenge to control the tuberculosis globally. The newer fluoroquinolones have clinical efficacy in some of the patients. So, it is needed the utility of new fluoroquinolone drugs for the treatment of tuberculosis.
\end{abstract}

Keywords: fluoroquinolones, levofloxacin, moxifloxacin, gatifloxacin, tuberculosis
Volume 2 Issue I - 2017

\author{
Mohammad Asif \\ Department of Pharmacy, Institute of Management and \\ Technology, India
}

\begin{abstract}
Correspondence: Mohammad Asif, Department of Pharmacy, GRD (PG) Institute of Management and Technology, Dehradun,
\end{abstract} 2480009, India, Email aasif32। @gmail.com

Received: November 23, 2016 | Published: January 26, 2017
Abbreviations: Fqs, fluoroquinolones; DS, drug susceptible; MDR, multidrug resistant; EBA, early bactericidal activity

\section{Introduction}

Quinolones are synthetic drugs synthesized by structural modification of the 4-oxo-1,4-dihydroquinolone nucleus or the 1,8-naphthyridone nucleus. Fluorination of these basic molecules, usually at position 6, resulted in the fluoroquinolones (FQs). Levofloxacin is the $\mathrm{S}(-)$-enantiomer of the parent racemic compound ofloxacin, whereas moxifloxacin and gatifloxacin are regarded as later generation C-8-methoxy FQs. The levofloxacin, moxifloxacin and gatifloxacin are newer FQs that have potent antituberculosis (anti$\mathrm{TB}$ ) activity, much of which is due to the C-8-methoxy moiety. ${ }^{1-3} \mathrm{~A}$ comprehensive review addressed the efficacy of FQs in TB, together with patient tolerability/safety, for the following indications-(i) firstline treatment of drug-susceptible (DS) pulmonary TB, (ii) first-line treatment of multidrug-resistant (MDR) TB and (iii) treatment of patients with intolerance to standard first-line anti-TB drugs. ${ }^{4}$ The data were insufficient to support the use of older FQs, especially ciprofloxacin, as substitute agents for DS or DR-TB. This review provides the role of FQs in the management of TB. ${ }^{5}$ The role of discussed FQs in treating TB is largely restricted to levofloxacin, moxifloxacin and gatifloxacin.

\section{FQs for the treatment of MDR-TB}

The MDR-TB has confirmed the dose-dependent efficacy of ofloxacin in the treatment of TB and the $800 \mathrm{mg}$ once-daily dose was found to be superior to the $300 \mathrm{mg}$ once-daily dose, achieving a more rapid and higher proportion of culture negativity. The use of FQs (ofloxacin and ciprofloxacin) for the treatment of MDR-TB has emerged ${ }^{6-9}$ with success rates usually around $70 \%$. A study on MDRTB has shown that the use of FQs was independently linked with improved initial microbiological outcome, as well as survival from all causes of death. ${ }^{10}$ The pivotal role of the FQs in the chemotherapy of MDR-TB.

The six standardized treatment regimens for MDR-TB (Figure 1), ${ }^{11}$ the most effective treatment regimen required a minimum duration of nine months with gatifloxacin, clofazimine, ethambutol, and pyrazinamide throughout, supplemented by prothionamide, kanamycin and high-dose isoniazid during an intensive phase of a minimum of four months, giving a relapse-free success rate of $87.9 \%$. The treatment success rate for the earlier ofloxacin-containing regimen was only $69.0 \%{ }^{12}$ The combination of amikacin, ethionamide, moxifloxacin and pyrazinamide has shown good efficacy. ${ }^{13}$ The use of moxifloxacin for MDR-TB, the treatment success rate was only $51.7 \% .{ }^{14}$ There was no clear report of the chemotherapy response rate for several patients with MDR-TB. ${ }^{15}$ The optimal duration of treatment for MDR-TB using a FQs-containing regimen is currently unknown. The successfully improved when the length of treatment was at least 18 months, and if patients received directly observed therapy throughout. ${ }^{16}$ However, some patients could be adequately treated with newer FQs for shorter periods to achieve a relapse-free cure.

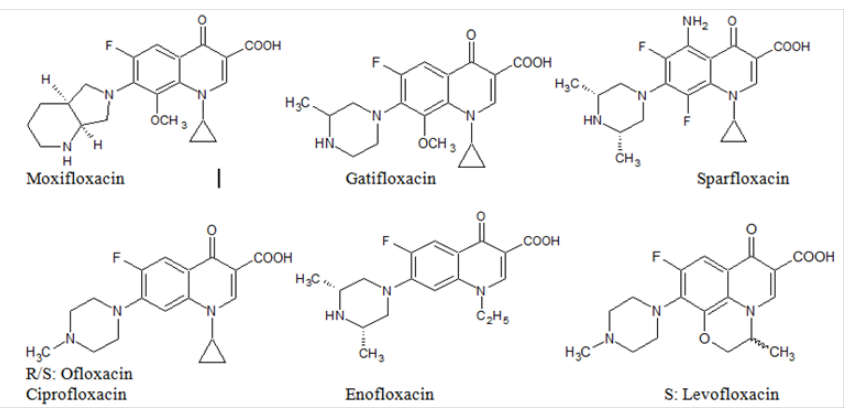

Figure I The six standardized treatment regimens for MDR-TB.

The FQ resistance in Mycobacterium tuberculosis (Mtb) can emerge following the injudicious use of this class of drugs, especially in the setting of MDR-TB, alongside the suboptimal use of accompanying drugs too few in numbers and/or too low a dosage. ${ }^{17,18}$ Poor drug quality can also be an issue. Overzealous use of FQs in the treatment of infections of the lower respiratory tract and other origins, might also contribute to the development of FQR-TB. ${ }^{19}$ As aminoglycosides/capreomycin also have potent anti-TB activity, the "loss" of these second-line injectable preparations together with FQs, through their suboptimal use in the treatment of MDR-TB, would result in the development of extensively drug-resistant (XDR) TB. ${ }^{20}$ This latter disease poses an even more "complicated" scenario of drug resistance than FQR, MDR-TB, and is generally linked with a treatment success rate of $50 \%$ or less. ${ }^{21}$ An analysis of the treatment outcomes and survival based on drug resistance patterns in MDR-TB 
strongly underscores the appropriateness of the definition XDR-TB and its association with a dismal prognosis. ${ }^{22}$

The potential usefulness of levofloxacin in treating DR-TB, ${ }^{23}$ a comparison between ofloxacin and levofloxacin ${ }^{24}$ has also revealed that the latter $\mathrm{FQ}$, when substituting for the former, in regimens with similar accompanying drugs, resulted in higher success rates for both ofloxacin-susceptible $(96.2 \%$ vs. $87.5 \%)$ and ofloxacinresistant (78.6\% vs. $45.5 \%)$ MDR-TB treatment. Thus, levofloxacin is quite likely to be more efficacious than ofloxacin when included in multidrug regimens for treating MDR-TB, including the "difficult" forms.

The C-8-methoxy FQs moxifloxacin and gatifloxacin might also have activity against ofloxacin-resistant $M t b$ isolates, including those that are MDR, notwithstanding the phenomenon of partial crossresistance among members of the FQ class. ${ }^{25,26}$ Indeed, these two newer FQs have lower mutant prevention concentrations for $M t b$ and should have a greater potential to restrict the development of bacillary resistance. ${ }^{27}$ However, it appears that for efficient suppression of development of DR-TB, high-dose moxifloxacin is preferable, but could well be limited by intolerability. ${ }^{28}$ In a meta analysis on the treatment outcomes of patients with XDR-TB, ${ }^{29} 43.7 \%$ exhibited a cure or treatment completion.

\section{Newer FQs for the treatment of DS-TB}

The most commonly encountered indication for the use of FQs in current practice is intolerance to standard first line anti-TB drugs, especially due to hepatic dysfunction. ${ }^{4}$ Although some patients can be satisfactorily returned to the originally scheduled first line drug regimen, most affected patients require the use of a relatively nonhepatotoxic regimen, on an interim or definitive basis. ${ }^{30}$ Earlier reports on this subject largely involved ofloxacin, used in conjunction with streptomycin and ethambutol. ${ }^{31}$ In case of definitive treatment of TB, ofloxacin/levofloxacin can be used together with isoniazid/ rifampicin, plus, perhaps, even low-dose pyrazinamide, depending on the liver reserve. ${ }^{31}$ A study on TB patients with liver injury, prescribed an alternative therapeutic regimen (streptomycin, ethambutol and ofloxacin) for three months followed by nine months of ethambutol and ofloxacin, this alternative regimen proved well tolerated by the patients and was effective in $85 \% .{ }^{32}$ In another study involving patients who developed hepatotoxicity to first line anti-TB drugs, the use of levofloxacin and moxifloxacin caused no additional hepatic insult, and allowed smooth normalization of liver transaminases similar to the control patients. ${ }^{33}$ Hepatotoxicity due to first-line anti-TB drugs has been found to be particularly frequent among patients with solidorgan transplants, ${ }^{34}$ perhaps largely due to immunocompromization and the toxicity of anti-rejection drugs. The use of regimens containing ofloxacin/levofloxacin was especially beneficial. Aside from good tolerance, the lack of drug interactions proved to be advantageous. Random regimens could not be used. ${ }^{35}$ Other serious intolerance to standard first-line anti-TB drugs is rare. Important examples include agranulocytosis, ${ }^{36}$ thrombocytopenia ${ }^{37}$ and renal failure. ${ }^{38}$

\section{Levofloxacin use in these patients has produced a good outcome}

Aside from intolerance to conventional anti-TB drugs, the newer FQs may find a place in increasing the efficacy of anti-TB drug regimens due to their potent activity. The ofloxacin/levofloxacin penetrates the pleural cavity better than rifampicin ( $>10$-fold) and, thus, helps to strengthen the first-line therapy for TB empyema, ${ }^{39}$ although such efficacy following the addition of a FQ to the treatment is lacking. Moxifloxacin-containing regimens demonstrated a greatly reduced time to culture conversion, ${ }^{40}$ and a short treatment with such a regimen produced a stable cure. ${ }^{41}$ Based on these findings, the significant sterilizing activity of moxifloxacin might enable a shortening of the length of therapy for drug-susceptible TB. A report from India suggested the potential usefulness of ofloxacin for shortening the length of treatment of DS-Tz. ${ }^{42}$ The addition of moxifloxacin to isoniazid, rifampicin and pyrazinamide did not affect the two-month sputum culture status, but there was increased activity at earlier time points. ${ }^{43}$ Using serial sputum colony counting by non-linear mixed effects modeling, moxifloxacin substitution for ethambutol appeared superior during the early phase of a bi-exponential fall in colony counts, but a significant and similar acceleration of bacillary elimination during the late phase occurred with both moxifloxacin and gatifloxacin. ${ }^{44}$ At eight weeks, culture conversion to negative occurred in $80 \%$ patients in the moxifloxacin group, compared with $63 \%$ patients in the ethambutol group. ${ }^{45}$ Substituting moxifloxacin for isoniazid only showed a non-significant effects meta-analysis and meta-regression showed that studies in which a higher proportion of patients received a later-generation FQ (levofloxacin, moxifloxacin or sparfloxacin) reported a higher proportion of favorable treatment outcomes. ${ }^{29}$ This is interesting because it seems that later-generation FQs could improve the treatment success of XDR-TB, even though drug susceptibility testing had demonstrated bacillary resistance to a representative FQ. This issue should be systematically evaluated in well designed clinical trials.

\section{Other issues regarding the use of newer fluoroquinolones in tuberculosis}

The commonest side effects of moxifloxacin use are GI disturbance and neurological dysfunction. ${ }^{46}$ In addition, there is preincrease in sputum culture conversion at week $8 .{ }^{46}$ Adding moxifloxacin to the four standard first-line anti-TB drugs shortened the time to culture conversion, and the culture conversion rate after six weeks of treatment rose from $61 \%$ to $82 \%{ }^{47}$ Substitution of moxifloxacin for isoniazid can reduce the current length of therapy of DS-TB to four months. The OFLOTUB consortium is also investigating a four-month regimen based on gatifloxacin. Although an early bactericidal activity (EBA) study has shown significant results with moxifloxacin in patients with pulmonary $\mathrm{TB},{ }^{48}$ the early and extended EBA of levofloxacin, alongside that of moxifloxacin and gatifloxacin, has shown that levofloxacin $1,000 \mathrm{mg}$ daily produced potent EBA comparable with that of isoniazid, and better than that of moxifloxacin and gatifloxacin. ${ }^{49}$ Weekly moxifloxacin and rifapentine has been shown to be more active than twice-weekly rifampicin and isoniazid in mouse tuberculosis model. ${ }^{50}$ Daily dosing of rifapentine cured the disease in three months or less. ${ }^{51} \mathrm{~A}$ controlled clinical trial using high-dose rifapentine and moxifloxacin (RIFAQUIN) is now in progress. The rate of any major adverse events in those who used levofloxacin (because of DR-TB or intolerance to first-line anti-TB drugs) was almost half that in those who received standard therapy. ${ }^{52}$ Furthermore, there was no difference between the levofloxacin and control arms with respect to central nervous system, gastrointestinal (GI) tract, skin or musculoskeletal related events when adjusted for the concomitant drugs. These findings strongly corroborate those from observational and other studies of the levofloxacin treatment of TB. However, it might be useful to remember some rare side effects related to ofloxacin/levofloxacin use including arthropathy, fungal superinfection and antibiotic-related colitis. ${ }^{53}$ 


\section{Safety/tolerance of newer FQs}

The beneficial use of moxifloxacin in treating TB in human immunodeficiency virus (HIV)-infected patients when conventional arrhoea was found to be modest after controlling for sex, age, other antibiotic use, serum albumin, duration of hospital stay and nasogastric feeding. ${ }^{54}$ Although the risk for potential cardiotoxicity is perhaps higher for moxifloxacin, compared with levofloxacin, ${ }^{55} \mathrm{a}$ randomized trial involving the cardiac rhythm safety of moxifloxacin versus levofloxacin in elderly patients with community-acquired pneumonia has shown them to have a comparable risk and safety. ${ }^{56}$ However, it is important to remember that this may not be the case when considering long-term use of these FQs in the treatment of TB. Extreme caution must thus be exercised in patients with underlying cardiac diseases or QTc prolongation, especially for those with risk factors for torsades de pointes. Gatifloxacin use is associated with GI and neurological adverse reactions like moxifloxacin. It also has potential cardiotoxicity. ${ }^{57}$ However, most importantly, it is associated with dysglycaemia, ${ }^{58}$ especially in older patients.

Despite the promise of the newer FQs in the future treatment of $\mathrm{TB}$, such optimism is somewhat tempered by the escalating rates of FQ resistance in $M t b$ in many parts of the world, especially in countries with a high incidence of TB. ${ }^{59}$ Empirical use of the newer FQs may also mask the diagnosis of TB, with a resultant delay in the start of treatment and a poor outcome. ${ }^{60}$ Another concern is the interaction of moxifloxacin and gatifloxacin with rifampicin, resulting in potential attenuation of the efficacy of the former $\mathrm{FQ},{ }^{61}$ and a potentially increased risk of toxicity for the latter FQ. ${ }^{62}$

\section{Conclusion}

The newer fluoroquinolones have good bactericidal and sterilizing activities against $M t b$. They could support the existing antibiotic armamentarium for the therapeutic control of drug-resistant and drugsusceptible tuberculosis. The potential adverse aspects associated with their use in the disease merit further exploration and evaluation in order to develop optimum regimens.

\section{Acknowledgements}

None.

\section{Conflict of interest}

The author declares no conflict of interest.

\section{References}

1. Fattorini L, Tan D, Iona E, et al. Activities of moxifloxacin alone and in combination with other antimicrobial agents against multidrug-resistant Mycobacterium tuberculosis infection in BALB/c mice. Antimicrob Agents Chemother. 2003;47(1):360-362.

2. Asif M. Study of clinically used and recently developed antimycobacterial agents. Orien Pharm \& Experi Med. 2012;12(1):15-34.

3. Asif MA. Recent advances in the development of salicylic acid analogues as anti-tubercular agents. Discovery Chemistry. 2021;12(13):1404-1418.

4. Moadebi S, Harder CK, Fitzgerald MJ, et al. Fluoroquinolones for the treatment of pulmonary tuberculosis. Drugs. 2007;67(14):2077-2099.

5. Asif M, Siddiqui AA, Husain A. Quinolone derivatives as antitubercular drugs. Medicinal Chemistry Research. 2013;22(3):1029-1042.

6. Yew WW, Chan $\mathrm{CK}$, Chau $\mathrm{CH}$, et al. Outcomes of patients with multidrug-resistant pulmonary tuberculosis treated with ofloxacin levofloxacin-containing regimens. Chest. 2000;117(3):744-751.
7. Tahaoğlu, Törün T, Sevim T, et al. The treatment of multidrug-resistant tuberculosis in Turkey. N Engl J Med. 2001;345(3):170-174.

8. Leimane V, Riekstina V, Holtz $\mathrm{TH}$, et al. Clinical outcome of individualised treatment of multidrug-resistant tuberculosis in Latvia: a retrospective cohort study. Lancet. 2005;365(9456):318-326.

9. Chiang CY, Enarson DA, Yu MC, et al. Outcome of pulmonary multidrug-resistant tuberculosis: a 6-yr follow-up study. Eur Respir J. 2006;28(5):980-985.

10. Chan ED, Laurel V, Strand MJ, et al. Treatment and outcome analysis of 205 patients with multidrug-resistant tuberculosis. Am J Respir Crit Care Med. 2004;169(10):1103-1119.

11. Van Deun A, Maug AK, Salim MA, et al. Short, highly effective, and inexpensive standardized treatment of multidrug-resistant tuberculosis. Am J Respir Crit Care Med. 2010;182(5):684-692.

12. Van Deun A, Salim MA, Das AP, et al. Results of a standardised regimen for multidrug-resistant tuberculosis in Bangladesh. Int J Tuberc Lung Dis. 2004;8(5):560-567.

13. Lounis N, Veziris N, Chauffour A, et al. Combinations of R207910 with drugs used to treat multidrug-resistant tuberculosis have the potential to shorten treatment duration. Antimicrob Agents Che-mother. 2006;50(11):3543-3547.

14. Codecasa LR, Ferrara G, Ferrarese M, et al. Long-term moxifloxacin in complicated tuberculosis patients with adverse reactions or resistance to first line drugs. Respir Med. 2006;100(9):1566-1572.

15. Valerio G, Bracciale P, Manisco V, et al. Long-term tolerance and effectiveness of moxifloxacin therapy for tuberculosis: preliminary results. $J$ Chemother. 2003;15(1):66-70.

16. Orenstein EW, Basu S, Shah NS, et al. Treatment outcomes among patients with multidrug-resistant tuberculosis: systematic review and meta-analysis. Lancet Infect Dis. 2009;9(3):153-161.

17. Wang JY, Lee LN, Lai HC, et al. Fluoroquinolone resistance in Mycobacterium tuberculosis isolates: associated genetic mutations and relationship to antimicrobial exposure. J Antimicrob Chemother. 2007;59(5):860-865

18. Caminero JA. Likelihood of generating MDR-TB and XDR-TB under adequate National Tuberculosis Control Programme implementation. Int J Tuberc Lung Dis. 2008;12(8):869-877.

19. Devasia RA, Blackman A, Gebretsadik T, et al. Fluoroquinolone resistance in Mycobacterium tuberculosis: the effect of duration and timing of fluoroquinolone exposure. Am J Respir Crit Care Med. 2009;180(4):365-370.

20. Migliori GB, Lange C, Centis R, et al. Resistance to second-line injectables and treatment outcomes in multidrug-resistant and extensively drug-resistant tuberculosis cases. Eur Respir J. 2008;31:1155-1159.

21. Kim HR, Hwang SS, Kim HJ, et al. Impact of extensive drug resistance on treatment outcomes in non-HIV-infected patients with multidrugresistant tuberculosis. Clin Infect Dis. 2007;45(10):1290-1295.

22. Kim DH, Kim HJ, Park SK, et al. Treatment outcomes and survival based on drug resistance patterns in multidrug-resistant tuberculosis. Am J Respir Crit Care Med. 2010;182(1):113-119.

23. Richeldi L, Covi M, Ferrara G, et al. Clinical use of levofloxacin in the long-term treatment of drug resistant tuberculosis. Monaldi Arch Chest Dis. 2002;57(1):39-43.

24. Yew WW, Chan CK, Leung CC, et al. Comparative roles of levofloxacin and ofloxacin in the treatment of multidrug-resistant tuberculosis: preliminary results of a retrospective study from Hong Kong. Chest. 2003;124(4):1476-1481 
25. Piersimoni C, Lacchini C, Penati V, et al. Validation of the agar proportion and 2 liquid systems for testing the susceptibility of Mycobacterium tuberculosis to moxifloxacin. Diagn Microbiol Infect Dis. 2007;57(3):283-287.

26. Devasia RA, Blackman A, May C, et al. Fluoroquinolone resistance in Mycobacterium tuberculosis: an assessment of MGIT 960, MODS and nitrate reductase assay and fluoroquinolone cross-resistance. $J$ Antimicrob Chemother. 2009;63(6):1173-1178.

27. Rodríguez JC, Cebrián L, López M, et al. Mutant prevention concentration: comparison of fluoroquinolones and linezolid with Mycobacterium tuberculosis. J Antimicrob Che-mother. 2004;53(3):441-444.

28. Gumbo T, Louie A, Deziel MR, et al. Selection of a moxifloxacin dose that suppresses drug resistance in Mycobacterium tuberculosis, by use of an in vitro pharmacodynamic infection model and mathematical modeling. In-fect Dis. 2004;190(9):1642-1651.

29. Jacobson KR, Tierney DB, Jeon CY, et al. Treatment outcomes among patients with extensively drug-resistant tuberculosis: systematic review and meta-analysis. Clin Infect Dis. 2010;51(1):6-14.

30. Saukkonen JJ, Cohn DL, Jasmer RM, et al. An official ATS statement: hepatotoxicity of antituberculosis therapy. Am J Respir Crit Care Med. 2006;174(8):935-952.

31. Saigal S, Agarwal SR, Nandeesh HP, et al. Safety of an ofloxacin-based antitubercular regimen for the treatment of tuberculosis in patients with underlying chronic liver disease: a preliminary report. $J$ Gastroenterol Hepatol. 2001;16(9):1028-1032.

32. Szklo A, Mello FC, Guerra RL, et al. Alternative anti-tuberculosis regimen including ofloxacin for the treatment of patients with hepatic injury. Int J Tuberc Lung Dis. 2007;11(7):775-780.

33. Ho CC, Chen YC, Hu FC, et al. Safety of fluoroquinolone use in patients with hepatotoxicity induced by anti-tuberculosis regimens. Clin Infect Dis. 2009;48(11):1526-1533.

34. Zhang XF, Lv Y, Xue WJ, et al. Mycobacterium tuberculosis infection in solid organ transplant recipients: experience from a single center in China. Transplant Proc. 2008;40(5):1382-1385.

35. Bonora S, Mondo A, Trentini L, et al. Moxifloxacin for the treatment of HIV-associated tuberculosis in patients with contraindications or intolerance to rifamycins. J Infect. 2008;57(1):78-81.

36. Shishido Y, Nagayama N, Masuda K, et al. Agranulocytosis due to anti-tuberculosis drugs including isoniazid (INH) and rifampicin (RFP)--a report of four cases and review of the literature. Kekkaku. 2003;78(11):683-689.

37. Onoda T, Murakami K, Eda R, et al. Rifampicin-induced severe thrombocytopenia in a patient with miliary tuberculosis. Kekkaku. 2003;78(7):491-496.

38. Amano H1, Takamori M, Fujita A, et al. A case of sternoclavicular joint tuberculosis with renal failure due to rifampicin. Kekkaku. 2009;84(8):591-595

39. Yew WW, Lee J. Drug treatment of chronic tuberculous empyema. Chest. 1992;101(6):1741-1742.

40. Nuermberger EL, Yoshimatsu T, Tyagi S, et al. Moxifloxacin-containing regimen greatly reduces time to culture conversion in murine tuberculosis Am J Respir Crit Care Med. 2004;169(3):421-426.

41. Nuermberger EL, Yoshimatsu T, Tyagi S, et al. Moxifloxacin-containing regimens of reduced duration produce a stable cure in murine tuberculosis. Am J Respir Crit Care Med. 2002;170(10):1131-1134.

42. Tuberculosis research centre (Indian Council of Medical Re-search) Indian J Tuberc. 49:27-38.

43. Burman WJ, Goldberg S, Johnson JL, et al. Moxifloxacin versus ethambutol in the first 2 months of treatment for pulmonary tuberculosis. Am J Respir Crit Care Med. 2006;174(3):331-338.
44. Rustomjee R, Lienhardt C, Kanyok T, et al. A Phase II study of the sterilising activities of ofloxacin, gatifloxacin and moxifloxacin in pulmonary tuberculosis. Int J Tuberc Lung Dis. 2008;12(2):128-138.

45. Conde MB, Efron A, Loredo C, et al. Moxifloxacin versus ethambutol in the initial treatment of tuberculosis: a double-blind, randomised, controlled phase II trial. Lancet. 2009;373(9670):1183-1189.

46. Dorman SE, Johnson JL, Goldberg S, et al. Substitution of moxifloxacin for isoniazid during intensive phase treatment of pulmonary tuberculosis. Am J Respir Crit Care Med. 2009;180(3):273-280.

47. Wang JY, Wang JT, Tsai TH, et al. Adding moxifloxacin is associated with a shorter time to culture conversion in pulmonary tuberculosis. Int $J$ Tuberc Lung Dis. 2010;14(1):65-71.

48. Gosling RD, Uiso LO, Sam NE, et al. The bactericidal activity of moxifloxacin in patients with pulmonary tuberculosis. Am J Respir Crit Care Med. 2003;168(11):1342-1345.

49. Johnson JL, Hadad DJ, Boom WH, et al. Early and extended early bactericidal activity of levofloxacin, gatifloxacin and moxifloxacin in pulmonary tuberculosis. Int J Tuberc Lung Dis. 2006;10(6):605-612.

50. Rosenthal IM, Williams K, Tyagi S, et al. Weekly moxifloxacin and rifapentine is more active than the denver regimen in murine tuberculosis. Am J Respir Crit Care Med. 2005;172(11):1457-1462.

51. Rosenthal IM, Zhang M, Williams KN, et al. Daily dosing of rifapentine cures tuberculosis in three months or less in the murine model. PLoS Med. 2007;4(12):e344.

52. Marra F, Marra CA, Moadebi S, et al. Levofloxacin treatment of active tuberculosis and the risk of adverse events. Chest. 2005;128(3):14061413.

53. Yew WW, Chau CH, Lee J. Superficial fungal infection of the skin during treatment of tuberculosis. Int J Tuberc Lung Dis. 2001;6(12:1132.

54. Chang KC, Leung CC, Yew WW, et al. Analyses of fluoroquinolones and Clostridium difficile-associated diarrhoea in tuberculosis patients. Int $J$ Tuberc Lung Dis. 2009;13(3):341-346.

55. Carbon C. Comparison of side effects of levofloxacin versus other fluoroquinolones. Chemotherapy. 2001;47(Supp1 3):9-14.

56. Morganroth J, Dimarco JP, Anzueto A, et al. A randomized trial comparing the cardiac rhythm safety of moxifloxacin vs levofloxacin in elderly patients hospitalized with community-acquired pneumonia. Chest. 2005;128(5):3398-3406.

57. Iannini PB. Cardiotoxicity of macrolides, ketolides and fluoroquinolones that prolong the QTc interval. Expert Opin Drug Saf. 2002;1:121-128.

58. Park Wyllie LY, Juurlink DN, KoppA, et al. Outpatient gatifloxacin therapy and dysglycemia in older adults. $N$ Engl J Med. 2016;354(13):13521361.

59. Agrawal D, Udwadia ZF, Rodri guez C, et al. Increasing incidence of fluoroquinolone-resistant Mycobacterium tuberculosis in Mumbai, India. Int J Tuberc Lung Dis. 2009;13(1):79-83.

60. Chang $\mathrm{KC}$, Leung $\mathrm{CC}$, Yew WW, et al. Newer fluoroquinolones for treating respiratory infection: do they mask tuberculosis? Eur Respir J. 2010;35(3):606-613

61. Nijland HM, Ruslami R, Suroto AJ, et al. Rifampicin reduces plasma concentrations of moxifloxacin in patients with tuberculosis. Clin Infect Dis. 2007;45(8):1001-1007.

62. McIlleron H, Norman J, Kanyok TP, et al. Elevated gatifloxacin and reduced rifampicin concentrations in a single-dose interaction study amongst healthy volunteers. J Antimicrob Chemother. 2007;60(6):13981401. 